\title{
Electronic logic gates from three-segment nanowires featuring two $\mathrm{p}-\mathrm{n}$ heterojunctions
}

\author{
Nan Chen ${ }^{1,2}$, Songhua Chen ${ }^{1,2}$, Canbin Ouyang ${ }^{1}$, Yanwen $\mathrm{Yu}^{1,2}$, Taifeng Liu ${ }^{1,2}$, Yongjun $\mathrm{Li}^{1}$, Huibiao Liu ${ }^{1}$ \\ and Yuliang $\mathrm{Li}^{1}$
}

\begin{abstract}
We report a novel nanodevice using $\pi$-conjugated polymers and inorganic semiconductor dual composite $\mathbf{P}-\mathrm{N}$ junctions single nanowire constructed by PEDOT-PbS-PPY (EPP) nanowire as a two-input OR gate. The nanodevices logic gate may show some key value in nanoelectronic components.
\end{abstract}

NPG Asia Materials (2013) 5, e59; doi:10.1038/am.2013.36; published online 16 August 2013

Keywords: $\pi$-conjugated; logic gate; organic/inorganic hybrid; $\mathrm{p}-\mathrm{n}$ heterojunctions; polymers

\section{INTRODUCTION}

With the rapid development of nanoelectronics, one-dimensional semiconductor nanostructures ${ }^{1-6}$ —including nanowires, ${ }^{7-9}$ nanotubes, ${ }^{10,11}$ nanofibers ${ }^{12,13}$ and nanocrystal, ${ }^{14,15}$ —are receiving increasing attention because their unique geometries (low dimensions and large surface-to-volume ratios) suggest many practical applications in electronics, photonics and optoelectronic devices. ${ }^{16,17} \pi$-conjugated polymers are organic semiconductors featuring delocalized $\pi$-electrons with a wide band gap that defines their affinity for electrons; they can be doped electrochemically to form $\mathrm{p}$ - or $\mathrm{n}$-type materials ${ }^{18,19}$ that behave as amorphous organic semiconductors. Because of their electronic conductivity, these materials are good candidates for developing diodes, optically responsive species, electrical on/off switches, photodetectors and chemical sensors. ${ }^{20-22}$ Nevertheless, conducting polymers are only single key elements in such systems - they must assemble with other materials to form the superstructures of the final devices. Combining the conducting polymers with different receptor units (for example, metals and inorganic semiconductors) can produce new composite materials exhibiting distinct architectures and properties not exhibited by their individual components. ${ }^{23,24}$ The $\mathrm{p}-\mathrm{n}$ junction is of great importance both in modern electronic applications and in our understanding of heterojunction - semiconductor interfaces and surfaces. The rational design of $\mathrm{p}-\mathrm{n}$ junctions can lead to the synthesis of new molecular materials. Recently, the self-assembly of $\mathrm{p}-\mathrm{n}$ junctions has led to interesting electrical and photoelectrical properties on the nanoscale. ${ }^{25}$ We are unaware, however, of any reports with respect to the fabrication of three-segment nanowires with dual $\mathrm{p}-\mathrm{n}$ heterojunctions as a new principle device for a logic gate-namely, devices featuring two diodes in series on two interfaces in an independent nanowire. Broadly speaking, the logic gate performs a logical operation on one or more logical inputs. We report herein the fabrication of heterojunction structures combining three kinds of semiconductors; they exhibit multifunctionality as logic circuits and display unusual performance in electronic devices.

\section{EXPERIMENTAL PROCEDURES}

\section{Materials}

3,4-Ethylenedioxythiophene (EDOT), tetrabutylammonium perchlorate, lithium perchlorate $\left(\mathrm{LiClO}_{4}\right)$, lead (II) chloride, sublimed sulfur, dimethyl sulfoxide, pyrole, acetonitrile and acetone were used in the synthesis process. All of the reagents were used as received. The anodic aluminum oxide (AAO) templates with a pore diameter of $200 \mathrm{~nm}$ and a thickness of $60 \mu \mathrm{m}$ were purchased from Whatman Co. (Shanghai, China). The nanowires were synthesized by a homemade electoral cell.

\section{Synthesis of PEDOT-PbS-PPY heterojunction nanowires}

The general synthetic procedure for the preparation of PEDOT-PbS-PPY heterojunction nanowires are as follows: first, evaporate a layer of Au on one side of the AAO template as conducting layer, and then put the AAO template into a homemade electrolytic cell as working electrode and an saturated calomel electrode (SCE) reference electrode in a three-electrode electrochemical cell. Poly(3,4-ethylenedioxythiophene) (PEDOT) deposition was carried out from a $10 \mathrm{~mm}$ EDOT and $0.2 \mathrm{M}$ tetrabutylammonium perchlorate acetonitrile solution by applying a voltage of $1.2 \mathrm{~V}$ (vs SCE) for an appropriate time (typically 2400 s). Thereafter, the AAO template containing PEDOT nanowires was used as a working electrode; $\mathrm{PbS}$ nanowires were deposited into the AAO template at a current density of $2.5 \mathrm{~mA} \mathrm{~cm}^{-2}$ in a dimethyl sulfoxide solution containing $28 \mathrm{mM} \mathrm{PbCl}_{2}$ and $95 \mathrm{~mm}$ element sulfur at a temperature between 100 and $105^{\circ} \mathrm{C}$; the template was then washed with hot dimethyl sulfoxide. In addition, polypyrrole (PPY) deposition was carried out from a $0.1 \mathrm{M}$ pyrole and $0.1 \mathrm{M} \mathrm{LiClO}_{4}$ acetonitrile solution by applying a voltage of $0.85 \mathrm{~V}$ (vs SCE) for an appropriate time (typically 3000 s). Finally, the PEDOT-PbS-PPY heterojunction nanowire-embedded AAO membrane was acquired. The AAO

${ }^{1}$ Beijing National Laboratory for Molecular Sciences (BNLMS), CAS Key Laboratory of Organic Solids, Institute of Chemistry, Chinese Academy of Sciences, Beijing, PR China and 2University of Chinese Academy of Sciences, Beijing, PR China

Correspondence: Professor Y Li or Dr H Liu, Beijing National Laboratory for Molecular Sciences (BNLMS), CAS Key Laboratory of Organic Solids, Institute of Chemistry, Chinese Academy of Sciences, Zhongguancun North First Street 2, Beijing 100190, PR China.

E-mail: ylli@iccas.ac.cn or liuhb@iccas.ac.cn

Received 19 October 2012; revised 13 June 2013; accepted 16 June 2013 
template was selectively etched by $\mathrm{NaOH}$ solution $(2 \mathrm{M})$ and cleaned by deionized water several times for latter analysis.

\section{Characterization}

Field emission scanning electron microscopy (SEM) images and energy dispersive $\mathrm{x}$-ray spectrometry (EDS) were taken from Hitachi S-4800 FESEM microscope at an accelerating voltage of 5 and $15 \mathrm{kV}$. Transmission electron microscopy images and selective-area electron diffraction patterns (SAED) were taken from JEOL JEM-1011 microscope at an accelerating voltage of $100 \mathrm{kV}$. Platinum microelectrodes $(200 \mathrm{~nm}$ in width, $300 \mathrm{~nm}$ in height) deposited by focus ion beam to construct the superstructure device of EPP nanowire (Figure $4 \mathrm{a}$ ), $\mathrm{PbS}$ nanowire and focus ion beam electrodes were made by Nova 200 NanoLab DualBeam-SEM/FIB system (Beijing, China). The electroproperty of devices were recorded with a Keithley 4200 SCS in a clean and shielded box at room temperature in air. The I-V curve of $\mathrm{PbS}$ nanowire with Pt electrodes (see Supplementary Figure S3) showed that it is an Ohmic contact between the individual $\mathrm{PbS}$ nanowire and Pt electrode.

\section{RESULTS AND DISCUSSION}

We used the p-type organic semiconductors PEDOT and PPy and the n-type inorganic semiconductor $\mathrm{PbS}$ to prepare our three-section axial heterojunction nanowire arrays through an associated approach of electrochemical deposition and template-directed growth.

Figure 1a presents a schematic model of an EPP (PEDOT-PbS$\mathrm{PPy}$ ) heterojunction nanowire array prepared using this combination process using the AAO template. SEM images revealed the morphology and dimensions of the EPP heterojunction nanowire arrays; for example, Figure $1 \mathrm{~b}$ presents a side-view image of a large cluster of EPP heterojunction nanowires. These well-defined nanowires feature smooth surfaces and diameters and lengths of $\sim 270 \mathrm{~nm}$ and $26 \mu \mathrm{m}$, respectively. In this image, the bottom portion of the EPP nanowires featured the PEDOT segments; these longest sections (nearly $11.2 \mu \mathrm{m}$ ) were the first to be grown during the EPP nanowire growth process (electrochemically under a minimum-resistance system) and were formed during a relatively short period of time. The brightest parts of

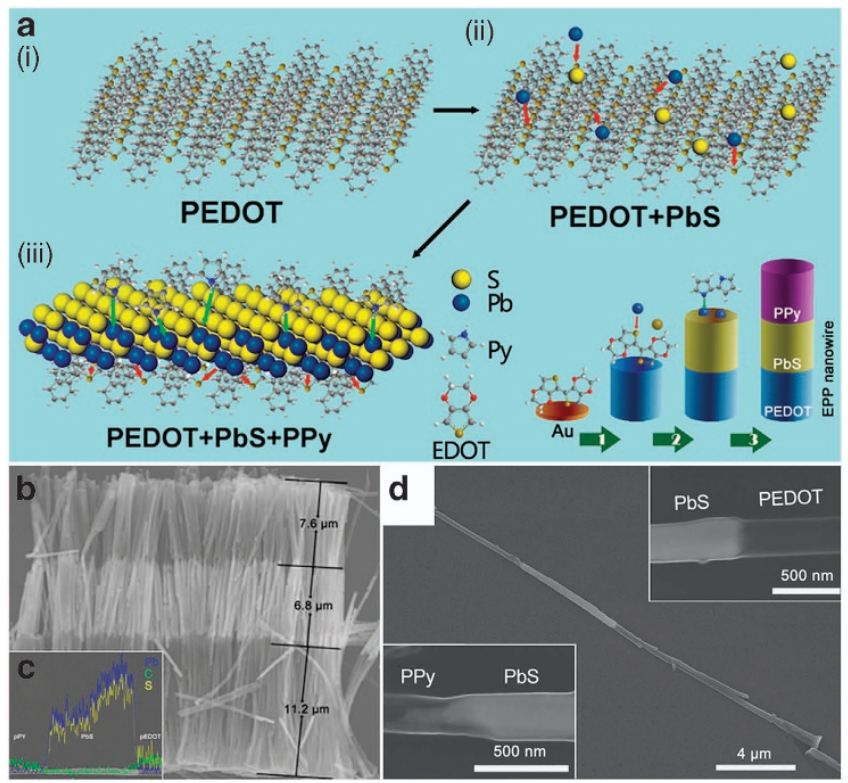

Figure 1 (a) Side-view SEM image of EPP heterojunction nanowire arrays after removal of the AAO template. (b) The linear scanning (SEM) of a single EPP heterojunction nanowire. (c) SEM image of an EPP heterojunction nanowire under low magnification; insets: higher-magnification images of its PbS/PEDOT and PPy/PbS p-n junctions. (d) Models of the growth process of the EPP heterojunction nanowire. the EPP nanowires, in the middle, were segments of the inorganic semiconductor $\mathrm{PbS}$ (length: ca. $6.8 \mu \mathrm{m}$ ). The top (third) section comprised PPy; the length of each PPy nanowire prepared under the largest resistance over a relatively long time was $\sim 7.6 \mu \mathrm{m}$ (see Supplementary Figure S1).

We confirmed the end-to-end structure of an as-prepared nanowire directly through element linear scanning (Figure 1c), which revealed the dispersion of the elements $\mathrm{C}$ (green), $\mathrm{S}$ (yellow) and $\mathrm{Pb}$ (blue). Energy dispersive X-ray spectrometry (EDS) of different parts of the hybrid nanowires clearly revealed the detailed chemical components, indicating that they were indeed composed sequentially of PEDOT, PPy and PbS. The SEM image of the single EPP nanowire in Figure 1d displays the three sections; the insets provide higher-magnification views of the two $\mathrm{p}-\mathrm{n}$ junctions (PbS/PEDOT, $\mathrm{PPy} / \mathrm{PbS}$ ). We observe that the single EPP heterojunction nanowire features clear interfaces, with good contact, between the organic and inorganic semiconductors; at the two junctions of the independent nanowire, the dark side is the organic semiconductor polymer (PPy or PEDOT) and the light side is the inorganic semiconductor (PbS). The good contact between the PbS segment and the PPy and PEDOT polymer segments arose from good matching of their structures and energies, as well as the strong coordinating ability of the heteroatoms of the organic moieties toward the metal ions of the inorganic semiconductor. In addition, these interactions increased the active surface area, suggesting good electronic contact between the inorganic and organic components. Together, these features result in the formation of two perfect interfaces in the independent heterojunction nanowire, allowing accurate measurement of the nanowire's physical properties.

We used transmission electron microscopy for further structural characterization of the EPP heterojunction nanowires. Figure 2a presents a typical image of a single EPP heterojunction nanowire; its diameter of $\sim 270 \mathrm{~nm}$ is consistent with that measured using SEM. The double organic/inorganic solid heterojunctions formed by the inorganic semiconductor $(\mathrm{PbS})$ and organic semiconductor (PEDOT and PPy) segments are clearly evident in the insets of Figure 2a, with $\mathrm{PbS}$ constituting the black parts and PEDOT and PPy the light parts. These interfaces were very clear, with the two solid components contacting tightly in each of the double heterojunctions. Highresolution TEM revealed the distinct interfacial structures of the PbS-PPy (Figure 2b) and PbS-PEDOT (Figure 2d) heterojunctions, indicating the crystallinity of the dark part PbS segments attached firmly to the amorphous light-colored parts PPy and PEDOT units. In each case, the lattice fringe spacing of $\mathrm{PbS}$ was $0.34 \mathrm{~nm}$ as shown in Figure $2 c$, suggesting the (111) plane of the hexagonal phase of a $\mathrm{PbS}$ crystal as the main growth direction for the $\mathrm{PbS}$ component of the nanowire. The SAED patterns of heterojunction nanowire (Figures $2 \mathrm{e}-\mathrm{g}$ ) for examining the crystallinity of the components on opposite sides of each interface confirmed the presence of a crystalline $\mathrm{PbS}$ nanowire and amorphous PPy and PEDOT segments.

We suggest the following three-step mechanism for the growth of the double $\mathrm{p}-\mathrm{n}$ junction EPP nanowire ${ }^{26}$ (Figure 1d): (i) EDOT molecules formed PEDOT nanowires through electropolymerization within the AAO template under an applied bias of $1.15 \mathrm{~V}$ (vs SCE). ${ }^{27}$ (ii) $\mathrm{The} \mathrm{Pb}^{2}+$ ions of $\mathrm{PbCl}_{2}$ absorbed strongly on the terminal surface of the PEDOT nanowire through weak coordinative interactions with the $\mathrm{S}$ atoms of the thiophene rings of PEDOT. Under a current density of $2.5 \mathrm{mAcm}^{-2}, \mathrm{~S}^{2-}$ anions were formed through the electrolysis of sulfur in dimethylformamide solution. The absorbed $\mathrm{Pb}^{2+}$ ions reacted with the $\mathrm{S}^{2-}$ anions to form $\mathrm{PbS}$ crystals on the surface of the PEDOT nanowire. The as-synthesized PbS crystals acted as nuclei for the formation of the $\mathrm{PbS}$ nanowire. We suspect that 


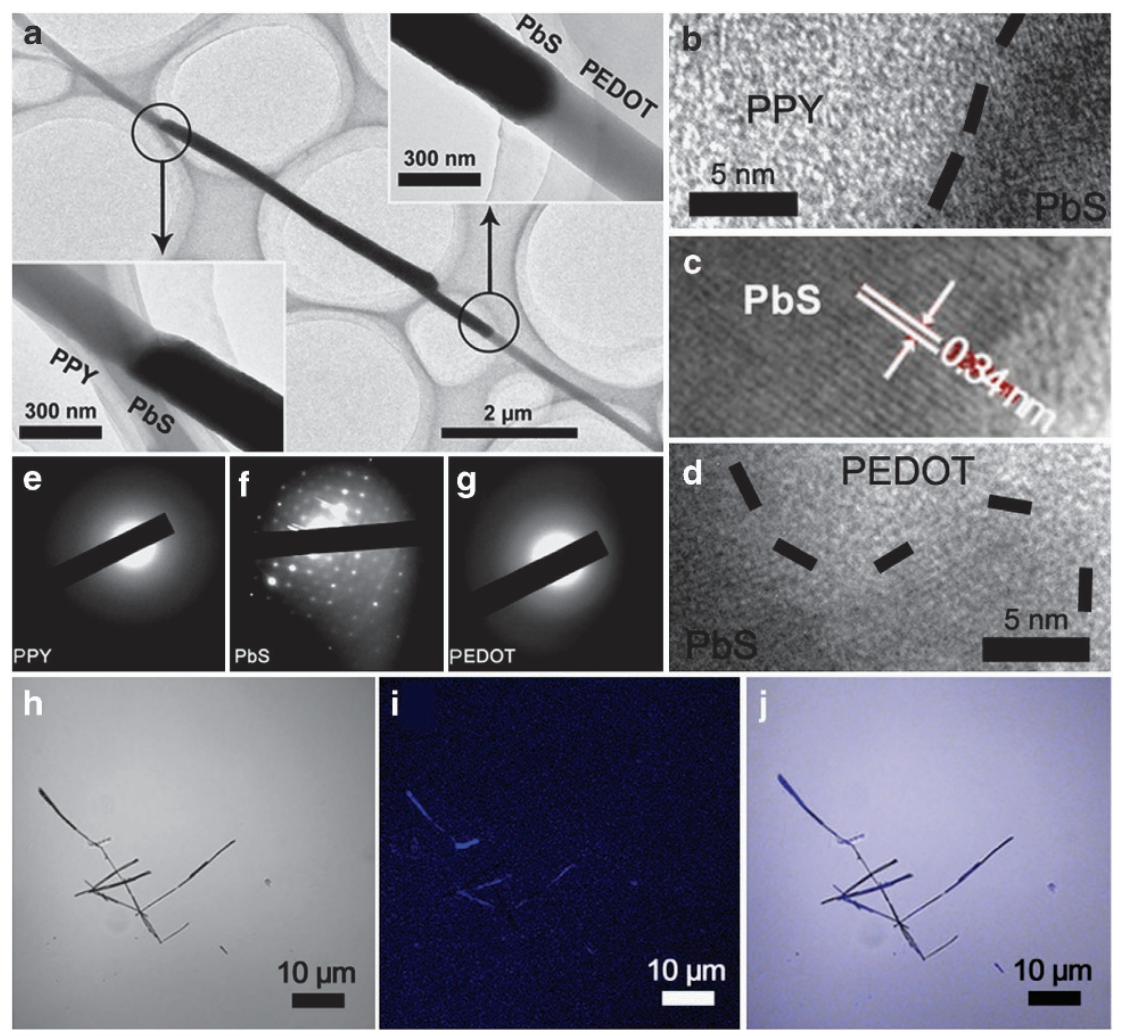

Figure 2 (a) TEM image of a single EPP heterojunction nanowire; insets: higher-magnification images of the corresponding PbS/PEDOT and PPy/PbS p-n junctions. (b, d) High-resolution TEM (HRTEM) images of the two p-n junction interfaces of the same EPP heterojunction nanowire. (c) HRTEM image of the PbS part of the EPP nanowire; the CdS has a lattice parameter of $0.34 \mathrm{~nm}$. (e-g) SAED patterns taken from segments in the single EPP heterojunction nanowire. (h) Optical image of several EPP heterojunction nanowires. (i) A fluorescence image of the same nanowires, removed from the alumina membrane. Confocal laser scanning microscopy (CLSM) image (j) of EPP heterojunction nanowires was merged by (h, i).

coordination of the $\mathrm{Pb}^{2+}$ ions and $\mathrm{S}$ atoms of the thiophene rings of PEDOT were responsible for the distinct interface evident in the highresolution TEM image in Figure 2b. (iii) Pyrrole molecules were adsorbed on the surface of the $\mathrm{PbS}$ nanowire through interactions between the $\mathrm{Pb}^{2+}$ ions and $\mathrm{N}$ atoms. The adsorbed pyrrole molecules were then polymerized under an applied bias of $0.85 \mathrm{~V}$ (vs SCE) at an approximate time. The distinct interface evident in the high-resolution TEM image in Figure 2d presumably resulted from the strong interactions between the $\mathrm{Pb}^{2+}$ ions and $\mathrm{N}$ atoms.

The structure of EPP heterojunction nanowire also can be confirmed by optical measurements according to the fluorescent properties of the three building blocks. In general, when the EPP heterojunction nanowire was excited with laser $(405 \mathrm{~nm})$, the $\mathrm{PbS}$ segment of the nanowire was blue, whereas there were no photoluminescence in the two organic segments as shown in the Figure $2 \mathrm{i}$.

Figure 3a presents a model of an EPP nanowire device (the SEM image of the single EPP heterojunction nanowire device made by focus ion beam was shown in Figure 4a and Supplementary Figure S2). The device comprised three segments: a blue part, PEDOT, defined herein as $\boldsymbol{A}$; a green part, $\mathrm{PbS}$, defined as $\boldsymbol{B}$; and a purple part, $\mathrm{PPy}$, defined as $\boldsymbol{F}$. Electrical measurements of the nanowire revealed that the PEDOT/PbS $\mathrm{p}-\mathrm{n}$ junction nanowire acted as a diode, exhibiting rectifying properties at room temperature (Figure 3c). Under a forward bias, the current increased upon increasing the applied bias; the turn-on voltage was greater than $5 \mathrm{~V}$. Under a reverse bias, the current transported through the junction nanowire was close to zero; the rectification ratio of the PbS-PEDOT part of the heterojunction nanowire diode was $\sim 289.1$ at a value of $V_{1-2 G}$ of $\pm 15 \mathrm{~V}$; the maximum current was $27 \mu \mathrm{A}$. The PbS-PPy part of the $\mathrm{p}-\mathrm{n}$ junction nanowire also exhibited diode behavior (Figure $3 \mathrm{~d}$ ), with conductivity greater than that of the PEDOT-PbS part; the rectification ratio of the PbS-PPy nanowire diode was $\sim 113.3$ at a value of $V_{2-3 G}$ of $\pm 15 \mathrm{~V}$; the maximum current was $70 \mu \mathrm{A}$. The electrical properties measured between $\boldsymbol{A}$ and $\boldsymbol{B}$ indicate that very low or negligible current was transported through the EPP nanowire from $\boldsymbol{A}$ to $\boldsymbol{B}$, and vice versa, when it had to pass through the two $\mathrm{p}-\mathrm{n}$ junctions; therefore, under either positive or negative bias conditions, segments $\boldsymbol{A}$ and $\boldsymbol{B}$ in the EPP nanowires were non-conducting in this case (Figure $3 b$ ). As a result, the axial EPP double $\mathrm{p}-\mathrm{n}$ junction threeterminal nanowire device functioned as a nano-logic two-input OR gate. ${ }^{28}$

To test the logic gate, we define the input value as $\mathbf{1}$ when the input voltage is higher than the turn-on voltage $(5 \mathrm{~V})$. In order to facilitate the testing, we first set the input voltage of $15 \mathrm{~V}$ as $\mathbf{1}$ (that is, higher than the diode turn-on voltage) (high level); we define an input voltage of $0 \mathrm{~V}$ or less than $0 \mathrm{~V}\left(V_{\mathrm{L} i} \in(-15,0)\right)$ as a $\mathbf{0}$ input voltage (low level), and we also set the current as the output signal; we define the output signal as 1 when the output current is higher than $10^{-5} \mathrm{~A}$, whereas in the other cases it is defined as $\mathbf{0}$. In detail, a $\mathbf{1}$ output high current from $\boldsymbol{F}$ results if one or both $(\boldsymbol{A}$ and $\boldsymbol{B})$ of the inputs to the gate are $\mathbf{1}$. If neither input is $\mathbf{1}$, a $\mathbf{0}$ output results such that the current is zero or very close to zero, as indicated in Figure 4c. The device behaves according to the truth table in Figure 4d. Therefore, the EPP nanowire is operated as a two-input OR logic gate. A two-input OR 

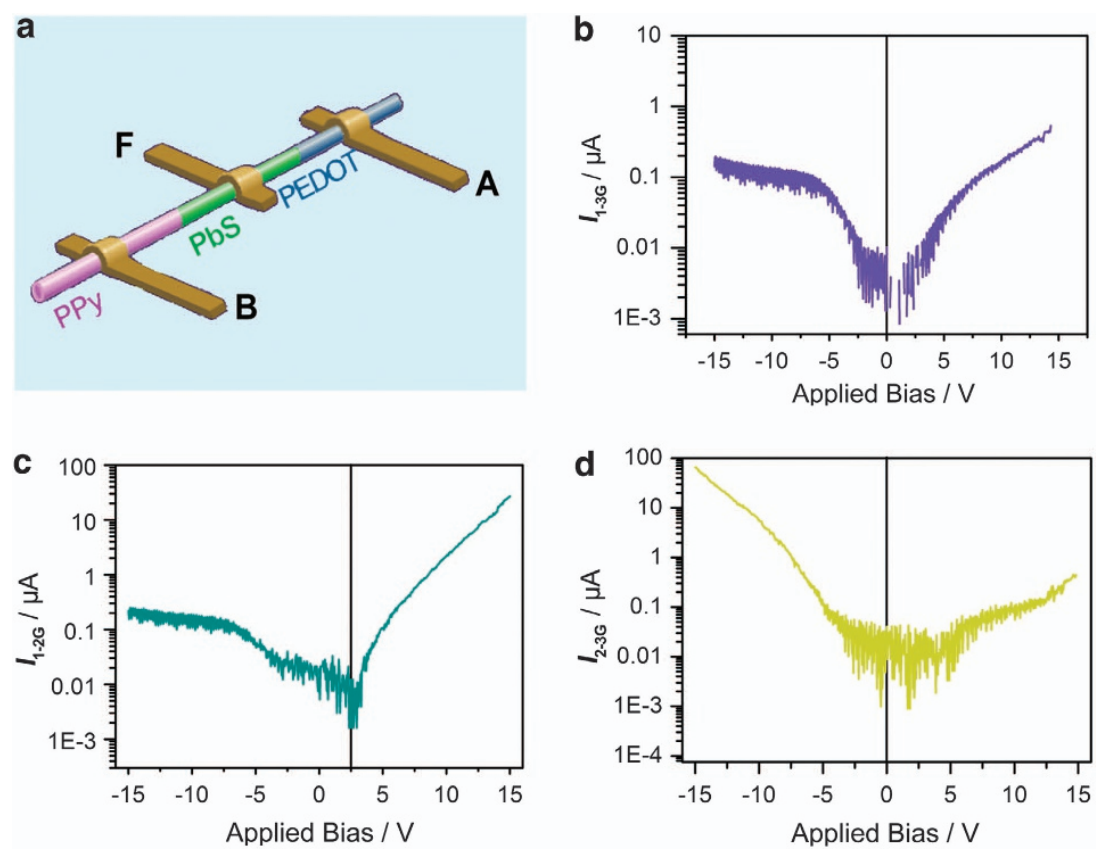

Figure 3 (a) The model of a single EPP heterojunction nanowire device. $I-V$ semi-log plot curves of the three-segment EPP nanodevices between (b) $\boldsymbol{A}$ and $\boldsymbol{B}$, (c) $\boldsymbol{A}$ and $\boldsymbol{F}$, (d) $\boldsymbol{B}$ and $\boldsymbol{F}$.
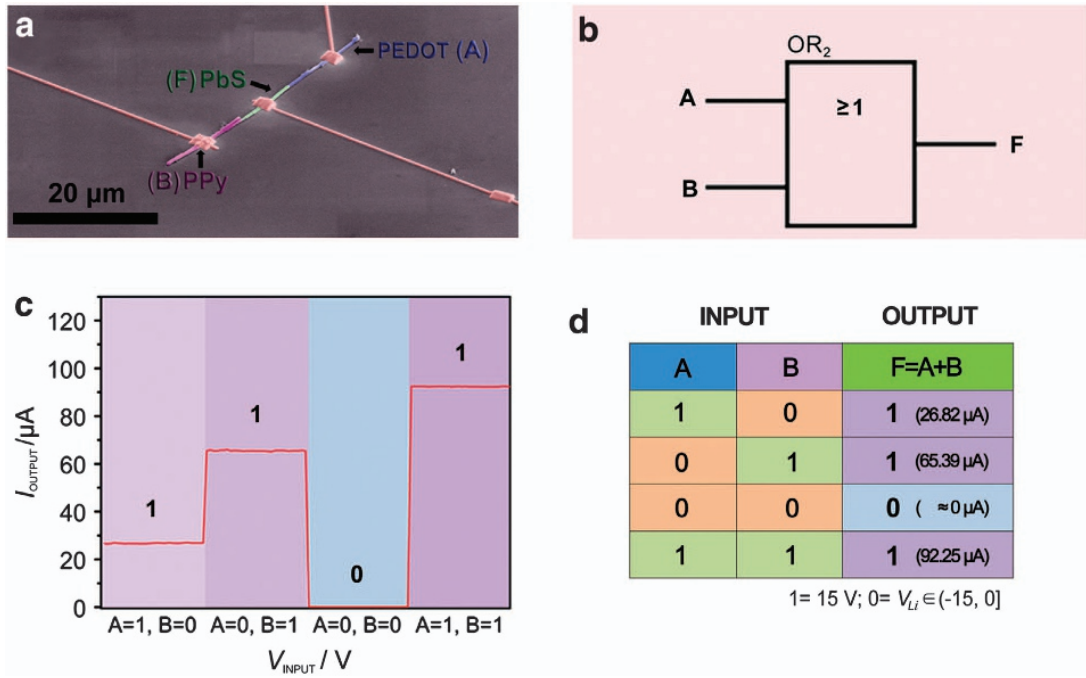

d

\begin{tabular}{|c|c|c|}
\multicolumn{2}{c}{ INPUT } & \multicolumn{1}{c|}{ OUTPUT } \\
\hline $\mathrm{A}$ & $\mathrm{B}$ & $\mathrm{F}=\mathrm{A}+\mathrm{B}$ \\
\hline 1 & 0 & $\mathbf{1}(26.82 \mu \mathrm{\mu})$ \\
\hline 0 & 1 & $\mathbf{1}(65.39 \mu \mathrm{A})$ \\
\hline 0 & 0 & $\mathbf{0}(\approx 0 \mu \mathrm{A})$ \\
\hline 1 & 1 & $\mathbf{1}(92.25 \mu \mathrm{A})$ \\
\hline \multicolumn{3}{|c|}{$1=15 \mathrm{~V} ; 0=V_{L i} \in(-15,0]$} \\
\hline
\end{tabular}

Figure 4 (a) SEM image of a single EPP heterojunction nanowire device made by focus ion beam. (b) Standard symbols for logic $O R_{2}$ gate (two-input OR gate). (c) The signal output of the logic two-input OR gate constructed using EPP nanowire, and (d) the output data of the logic two-input OR gate constructed using EPP nanowire.

gate is a digital logic gate that implements logical disjunction ${ }^{29}$ as displayed in Figure 4b.

\section{CONCLUSION}

In summary, we have fabricated a novel heterojunction structure combining one inorganic and two organic semiconductors. The different segments exhibited distinct self-assembly behavior, allowing us to control the production of one-dimensional three-segment nanowires featuring two diodes in series on two interfaces; the isolated organic/inorganic semiconductor wires displayed the novel properties of a two-input OR logic gate. We suspect that these devices might have applicability for fundamental research in the field of nanoscience and to applications in the field of nanotechnology, with great potential to produce new molecular electronic devices.

\section{DEDICATION}

This paper is dedicated to Professor Xiao-Zeng You for his 80th birthday.

\section{ACKNOWLEDGEMENTS}

This study was supported by the National Basic Research 973 Program of China (2011CB932302 and 2012CB9329001 and the National Nature Science Foundation of China (201031006, 91227113 and 21021091). 
1 Huang, Y., Duan, X. F., Wei, Q. Q. \& Lieber, C. M. Directed assembly of onedimensional nanostructures into functional networks. Science 291, 630-633 (2001).

2 Liu, H. B., Xu, J. L., Li, Y. J. \& Li, Y. L. Aggregate nanostructures of organic molecular materials. Acc. Chem. Res. 43, 1496-1508 (2010).

3 Lu, G., Li, S. Z., Guo, Z., Farha, O. K., Hauser, B. G., Qi, X. Y., Wang, Y., Wang, X., Han, S. Y., Liu, X. G., DuChene, J. S., Zhang, H., Zhang, Q. C., Chen, X., Ma, D. J., Loo, S. C. J., Wei, W. D., Yang, Y. H., Hupp, J. T. \& Huo, F. W. Imparting functionality to a metal-organic framework material by controlled nanoparticle encapsulation. Nature Chem. 4, 310-316 (2012).

4 Devan, R. S., Patil, R. A., Lin, J. -H. \& Ma, Y. -R. One-dimensional metal-oxide nanostructures: recent developments in synthesis, characterization, and applications. Adv. Funct. Mater. 22, 3326-3370 (2012)

5 Zheng, H. Y., Li, Y. J., Liu, H. B., Yin, X. D. \& Li, Y. L. Construction of heterostructure materials toward functionality. Chem. Soc. Rev. 40, 4506-4524 (2011).

6 Chen, N., Qian, X. M., Lin, H. W., Liu, H. B. \& Li, Y. L. Growing uniform copolymer nanowire arrays for high stability and efficient field emission. J. Mater. Chem. 22, 11068-11072 (2012)

7 Morrow, T. J., Li, M. W., Kim, J., Mayer, T. S. \& Keating, C. D. Programmed assembly of DNA-coated nanowire devices. Science 323, 352 (2009).

8 Chen, N., Qian, X. M., Lin, H. W., Liu, H. B., Li, Y. J. \& Li, Y. L. Synthesis and characterization of axial heterojunction inorganic-organic semiconductor nanowire arrays. Dalton Trans. 40, 10804-10808 (2011).

9 Chattopadhyay, S., Chen, L. -C. \& Chen, K. -H. Energy production and conversion applications of one-dimensional semiconductor nanostructures. NPG Asia Materials 3 , 74-81 (2011).

10 Wu, J., Gerstandt, K., Zhang, H. B., Liu, J. \& Hinds, B. J. Electrophoretically induced aqueous flow through single-walled carbon nanotube membranes. Nature Nanotech. 7, 133-139 (2012).

11 Bachtold, A., Hadley, P., Nakanishi, T. \& Dekker, C. Logic Circuits with Carbon Nanotube Transistors. Science 1317-1320 (2001).

12 Kjelstrup-Hansen, J., Hansen, O., Rubahn, H. -G. \& Bøggild, P. Mechanical properties of organic nanofibers. Small 2, 660-666 (2006).

13 Weitz, R. T., Harnau, L., Rauschenbach, S., Burghard, M. \& Kern, K. Polymer nanofibres via nozzle-free centrifugal spinning. Nano Lett. 8, 1187-1191 (2008).

14 Sun, S. H., Murray, C. B., Weller, D., Folks, L. \& Moser, A. Monodisperse FePt Nanoparticles and Ferromagnetic FePt Nanocrystal Superlattices. Science 287, 1989-1992 (2000).

15 Choi, J. J., Lim, Y. -F., Santiago-Berrios, M. B., Oh, M., Hyun, B. -R., Sun, L. F. Bartnik, A. C., Goedhart, A., Malliaras, G. G., Abruña, H. D., Wise, F. W. \& Hanrath, T. PbSe nanocrystal excitonic solar cells. Nano Lett. 9, 3749-3755 (2009).

$16 \mathrm{Li}$, J., Zhang, Y. L., To, S., You, L. D. \& Sun, Y. Effect of nanowire number, diameter, and doping density on nano-FET biosensor sensitivity. ACS Nano 8, 6661-6668 (2011).
17 Hochbaum, A. I. \& Yang, P. D. Semiconductor nanowires for energy conversion. Chem. Rev. 110, 527-546 (2010).

18 Hamedi, M., Herland, A., Karlsson, R. H. \& Inganäs, O. Electrochemical devices made from conducting nanowire networks self-assembled from amyloid fibrils and alkoxysulfonate PEDOT. Nano Lett. 8, 1736-1740 (2008).

19 Alam, M. M., Wang, J., Guo, Y. Y., Lee, S. P. \& Tseng, H. -R. Electrolyte gated transistors based on conducting polymer nanowire junction arrays. J. Phys. Chem. B 109, 12777-12784 (2005).

20 Gutsche, C., Niepelt, R., Gnauck, M., Lysov, A., Prost, W., Ronning, C. \& Tegude, F.-J. Direct determination of minority carrier diffusion lengths at axial GaAs nanowire $p-n$ junctions. Nano Lett. 12, 1453-1458 (2012).

21 Protasenko, V., Gordeyev, S. \& Kuno, M. Spatial and intensity modulation of nanowire emission induced by mobile charges. J. Am. Chem. Soc. 129, 13160-13171 (2007).

22 Kreno, L. E., Leong, K., Farha, O. K., Allendorf, M., Van Duyne, R. P. \& Hupp, J. T. Metal-organic framework materials as chemical sensors. Chem. Rev. 112, 1105-1125 (2012).

23 Xin, H., Reid, O. G., Ren, G. Q., Kim, F. S., Ginger, D. S. \& Jenekhe, S. A. Polymer nanowire/fullerene bulk heterojunction solar cells: how nanostructure determines photovoltaic properties. ACS Nano 4, 1861-1872 (2010).

24 Hsiao, C. -T. \& Lu, S. -Y. Morphological modulation of optoelectronic properties of organic-inorganic nanohybrids prepared with a one-step co-fed chemical vapor deposition polymerization process. J. Mater. Chem. 19, 6766-6772 (2009).

25 Chen, Y., Crittenden, J. C., Hackney, S., Sutter, L. \& Hand, D. W. Preparation of a novel $\mathrm{TiO}_{2}$-based p-n junction nanotube photocatalyst. Environ. Sci. Technol. 39, 1201-1208 (2005).

26 Guo, Y. B., Zhang, Y. J., Liu, H. B., Lai, S. W., Li, Y. L., Li, Y. J., Hu, W. P., Wang, S., Che, C. M. \& Zhu, D. B. Assembled organic/inorganic $p-n$ junction interface and photovoltaic cell on a single nanowire. J. Phys. Chem. Lett. 1, 327-330 (2010).

27 Groenendaal, L. B., Jonas, F., Freitag, D., Pielartzik, H. \& Reynolds, J. R. Poly(3,4ethylenedioxythiophene) and its derivatives: Past, present, and future. Adv. Mater. 12, 481-494 (2000).

28 Xia, F., Zuo, X. L., Yang, R. Q., White, R. J., Xiao, Y., Kang, D., Gong, X., Lubin, A. A., Vallée-Bélisle, A., Yuen, J. D., Hsu, B. Y. B. \& Plaxco, K. W. Label-free, dual-analyte electrochemical biosensors: A new class of molecular-electronic logic gates. J. Am. Chem. Soc. 132, 8557-8559 (2010).

29 Seelig, G., Soloveichik, D., Zhang, D. Y. \& Winfree, E. Enzyme-free nucleic acid logic circuits. Science 314, 1585-1588 (2006).

cc) (1) $(9)$ This work is licensed under a Creative Commons NC ND Attribution-NonCommercial-NoDerivs 3.0 Unported License. To view a copy of this license, visit http://creativecommons. org/licenses/by-nc-nd/3.0/

Supplementary Information accompanies the paper on the NPG Asia Materials website (http://www.nature.com/am) 